\title{
Functional Renormalization Group approach to the Kraichnan model
}

\author{
Carlo Pagan ${ }^{*}$ \\ Institut für Physik (WA THEP) Johannes-Gutenberg-Universität, Staudingerweg 7, 55099 Mainz, Germany
}

\begin{abstract}
We study the anomalous scaling of the structure functions of a scalar field advected by a random Gaussian velocity field, the Kraichnan model, by means of Functional Renormalization Group techniques. We analyze the symmetries of the model and derive the leading correction to the structure functions considering the renormalization of composite operators and applying the operator product expansion.
\end{abstract}

\section{INTRODUCTION}

Understanding the scaling of the velocity structure functions in turbulent flows is a challenging problem. Such scaling is known to be universal in the inertial range and shows small departures from the behaviour predicted by Kolmogorov [1]. To make progress in understanding the anomalous scaling of the structure functions a model for an advected passive scalar field, the Kraichnan model 2, turned out to be illuminating (similar models were considered long time ago by Corssin and Obukhov [3, 4]). One of the features which makes this model appealing is that the corrections to the canonical scaling of the structure functions can be analytically computed considering the zero modes of some operator $\mathcal{M}_{n}^{*}$ which acts on the equal time correlation functions of the scalar field [5, 6]. This result has been obtained via various methods among which the expansion in the Hölder exponent appearing in the covariance of the velocity field (i.e.: the $\varepsilon$ appearing in equation (2), an expansion for large spatial dimension and the Batchelor limit ( $\varepsilon$ close to 2 ) [5] 9. Furthermore the zero modes have been understood as statistical conservation laws [10. For a review of the results and a complete discussion we refer to [11. In this work we study the scaling of the structure functions of the Kraichnan model by means of the Functional Renormalization Group which offers the advantage of being a very adaptable framework.

The Renormalization Groups proved to be ideal to understand the anomalous scaling in statistical systems but the applications of its inherent concepts go far beyond. The Functional Renormalization Group (FRG) is a functional realization of the Wilsonian renormalization program and is applied successfully to many problems regarding out of equilibrium systems, for a review see 12 and [13. In the FRG framework one considers a scale dependent effective action, the Effective Average Action (EAA), which interpolates between the classical action and the standard effective action 14-16. Within this framework the issue of a fixed point for the functional integral associated to the randomly stirred Navier-Stokes equation has been first considered in [17] and more recently in [18] and [19, 20]. In particular in [20] a fixed point solution of the FRG equation associated to this problem has been investigated in $d=2$ and $d=3$. Nevertheless there is not still a quantitative prediction for the anomalous exponent of the structure functions and it may turn useful to address this question in a simpler setting. Let us stress that the FRG has been proved to be a solid framework in similar problems such as the scaling exponents of the Kardar-Parisi-Zhang equation and its correlation functions in the strong coupling regime [21, 22] (see [23] for the Burgers' equation).

The RG analysis of the Kraichnan model has been performed in a perturbative framework by means of an $\varepsilon$-expansion which is very similar to the one used in the analysis of critical phenomena [24]. The $\varepsilon$-expansion framework has been applied also to many other problems ranging from the randomly stirred Navier-Stokes equation to magnetic hydrodynamics, we refer to [25] and [26] for a complete list of references. Actually this framework turned out to be very efficient for the Kraichnan model and the anomalous exponents of the structure function have been computed up to order $O\left(\varepsilon^{3}\right)$ [27]. The inverse and the Wilsonian RG and their connection to the zero modes results have been studied in 28]. In this work we analyze the FRG approach to the Kraichnan model; this allows us to investigate a number of issues in a simpler setting and shows that the FRG is a viable framework for the computation of the anomalous exponents for the structure functions. Thus the main purpose of this work is to study in a concrete example the techniques required to understand the scaling of the structure functions in the FRG framework and in particular the role of composite operators. The paper is organized as follows: in the section [I] we introduce the path integral formalism for the model. In section III we consider the relevant RG equations and show how to obtain the latter ones within the FRG approach. In section IV] we study the IR fixed point of the model while in section $\mathrm{V}$ we consider the anomalous dimension of the relevant composite operators. In section VI we put together our results and discuss the scaling of the structure functions. In the final section we summarize our findings and consider possible outlooks.

* capagani@uni-mainz.de 


\section{PATH INTEGRAL FORMULATION OF THE KRAICHNAN MODEL}

In this section we recall the definition of the Kraichnan model and derive its path integral representation. The symmetries of the model are described in appendix A together with some non-renormalization theorems which follow. Let us consider a scalar quantity $\theta(t, x)$, e.g.: the temperature, which is advected by a fluid having random velocity $v(t, x)$. The equation for the dynamics of $\theta(t, x)$ is

$$
\partial_{t} \theta+v^{i} \partial_{i} \theta-\frac{\kappa}{2} \partial^{2} \theta=f
$$

where $\kappa$ is the molecular diffusivity and $f$ is a stochastic forcing term with Gaussian statistic and zero average. Note that equation (1) is a stochastic differential equation with multiplicative noise and is interpreted in the Stratonovich sense. More precisely the covariance of the forcing term is:

$$
\begin{aligned}
\left\langle f\left(t_{1}, x_{1}\right) f\left(t_{2}, x_{2}\right)\right\rangle & =\delta\left(t_{1}-t_{2}\right) F\left(\frac{x_{1}-x_{2}}{L_{F}}\right) \\
& \equiv D_{\bar{\theta}}
\end{aligned}
$$

where $L_{F}$ is the characteristic scale of the forcing (eventually we will denote $\left.L_{F}^{-1}=M\right)$. The random velocity $v(t, x)$ is also Gaussian and has zero average:

$$
\begin{aligned}
\left\langle v_{i}\left(t_{1}, x_{1}\right) v_{j}\left(t_{2}, x_{2}\right)\right\rangle & =\frac{D_{0}}{(2 \pi)^{d}} \delta\left(t_{1}-t_{2}\right) \int d^{d} k e^{i k x_{1}-i k x_{2}} \\
& \frac{P_{i j}}{\left(k^{2}+m^{2}\right)^{\frac{d}{2}+\frac{\varepsilon}{2}}} \\
& \equiv D_{v}
\end{aligned}
$$

where

$$
P_{i j}=\delta_{i j}-\frac{k_{i} k_{j}}{k^{2}}
$$

is the projector onto the transversal components (the fluid is incompressible). Throughout this work we assume the Stratonovich discretization prescription for all the relavant formulas unless otherwise stated. Note that the mass $m$ in the denominator avoids IR divergences and is a further scale besides $L_{F}$, which appears in the force correlator. Actually in the inertial regime the structure functions do not depend on $m$ and we set $m$ to zero (if one wanted to it is easy to keep this parameter). We will not need to introduce $m$ at all since the FRG framework is safe from IR divergences by construction.

Averages can be computed via the following path integral:

$$
Z=\int \mathcal{D} f \mathcal{D} v \mathcal{D} \theta P_{f f} P_{v v} \delta\left(\theta-\theta_{0}\right)
$$

where $\theta_{0}$ is a solution of equation (1) and $P_{f f}$ and $P_{v v}$ are the Gaussian weighting factors of $f$ and $v$ respectively.
We have:

$$
\begin{aligned}
Z= & \int \mathcal{D} f \mathcal{D} v \mathcal{D} \theta P_{f f} P_{v v} \delta\left(\partial_{t} \theta+v^{i} \partial_{i} \theta-\frac{\kappa}{2} \partial^{2} \theta-f\right) \\
& \left\|\operatorname{det}\left(\partial_{t}+v^{i} \partial_{i}-\frac{\kappa}{2} \partial^{2}\right)\right\|
\end{aligned}
$$

where the determinant comes from the change of variables in the path integral. For the time being we shall neglect this determinant and we will come back to it in a moment:

$$
\begin{aligned}
Z= & \int \mathcal{D} f \mathcal{D} v \mathcal{D} \theta e^{-\frac{1}{2} \int f\langle f f\rangle^{-1} f} e^{-\frac{1}{2} \int v\langle v v\rangle^{-1} v} \\
& \delta\left(\partial_{t} \theta+v^{i} \partial_{i} \theta-\frac{\kappa}{2} \partial^{2} \theta-f\right) \\
= & \int \mathcal{D} \bar{\theta} \mathcal{D} f \mathcal{D} v \mathcal{D} \theta e^{-\frac{1}{2} \int f\langle f f\rangle^{-1} f} e^{-\frac{1}{2} v\langle v v\rangle^{-1} v} \\
& \exp \left[i \int \bar{\theta}\left(\partial_{t} \theta+v^{i} \partial_{i} \theta-\frac{\kappa}{2} \partial^{2} \theta-f\right)\right] \\
= & \int \mathcal{D} \bar{\theta} \mathcal{D} v \mathcal{D} \theta e^{+\frac{1}{2} \int \bar{\theta}\langle f f\rangle \bar{\theta}} e^{-\frac{1}{2} \int v\langle v v\rangle^{-1} v} \\
& \exp \left[-\int \bar{\theta}\left(\partial_{t} \theta+v^{i} \partial_{i} \theta-\frac{\kappa}{2} \partial^{2} \theta\right)\right]
\end{aligned}
$$

In the last line we redefined the field $\bar{\theta}$ via $\bar{\theta} \rightarrow i \bar{\theta}$. The determinant in equation (4) is very similar to the one encountered in the path integral representation of the Langevin equation, see for instance [29] and references therein. Such determinant depends on the convention chosen for Heaviside theta function in zero: if $\theta(0)=0$ we are considering the Ito calculus while if $\theta(0)=1 / 2$ we are considering Stratonovich. In particular, as shown in 28], using Ito discretization prescription the determinant does not enter in the path integral and the action has the same form of the action $S_{\theta}$ defined in equation (5) below but with a different molecular diffusivity. As we already said in this work we will follow Stratonovich discretization prescription and we shall include the determinant contribution in the action via two Grassmann odd fields which we refer to as ghosts. Such choice may seem somehow more complicated since it doubles the number of fields. Nevertheless we would like to keep such ghost fields since they carry a physical and geometrical meaning in the path integral approach to classical mechanics [30, 31] and make manifest the supersymmetry in stochastic processes, see 32 and references therein. The ghost action associated to the determinant is simply:

$$
S_{g h}=\int \bar{c}\left(\partial_{t}+v^{i} \partial_{i}-\frac{\kappa}{2} \partial^{2}\right) c
$$

The full action is thus composed of two parts: $S_{g h}$ and

$$
S_{\theta}=\int\left[\bar{\theta}\left(\partial_{t}+v^{i} \partial_{i}-\frac{\kappa}{2} \partial^{2}\right) \theta-\frac{1}{2} \bar{\theta} D_{\bar{\theta}} \bar{\theta}+\frac{1}{2} v D_{v}^{-1} v\right](5)
$$

Note that the field $\bar{\theta}$ is the response field present in the Martin-Siggia-Rose formalism [33, see also [34, 35, and 
that $S_{\theta}$ and $S_{g h}$ are very similar due to the fact that equation (1) is linear in $\theta$. Thus the stochastic problem in equation (1) has been mapped to the field theory coming from expression (4). Now we are able to exploit the techniques available for standard field theory and in particular we will employ the Functional Renormalization Group discussed in the next section. Finally let us recall the dimension of the various quantities entering in the action, we have $[\theta]=T^{1 / 2},[\theta]=L^{-d} T^{-1 / 2},\left[D_{0}\right]=\frac{L^{2}}{T} L^{-\varepsilon}$ and $[\kappa]=\frac{L^{2}}{T}$. Following [24] we define $g=D_{0} / \kappa$ which has dimensions $[g]=L^{-\varepsilon}$.

\section{FUNCTIONAL RENORMALIZATION GROUP FOR THE KRAICHNAN MODEL}

Functional Renormalization Group provides a non perturbative framework for the implementation of the Wilsonian integration of high momentum modes. For a general introduction we refer the reader to the following list of reviews [37 41]. We will work via the Effective Average Action (EAA) which is a scale dependent generalization of the standard effective action. One first define a modified generating functional of connected Green's function $W_{k}$ :

$$
e^{W_{k}[J]}=\int \mathcal{D} \chi e^{-S-\Delta S_{k}+J \chi}
$$

where $\Delta S_{k}$ suppresses the integration of momentum modes $p^{2}<k^{2}$ and is quadratic in the fields with a kernel $R_{k}$, i.e.: $\Delta S_{k}=\frac{1}{2} \int \chi R_{k} \chi$. Note that this acts like an infrared cutoff. Thus let $\tilde{\Gamma}_{k}$ be the Legendre transform of $W_{k}$ and define the EAA subtracting the cutoff action which we added at the beginning:

$$
\Gamma_{k} \equiv \tilde{\Gamma}_{k}-\Delta S_{k}
$$

The $k$-dependence of the functional $\Gamma_{k}$ satisfy the following exact equation [14 16]

$$
\partial_{t} \Gamma_{k}=\frac{1}{2} \operatorname{Tr}\left[\left(\Gamma_{k}^{(2)}+R_{k}\right)^{-1} \partial_{t} R_{k}\right]
$$

where $\partial_{t}=k \partial_{k}$ is the logarithmic derivative with respect to the cutoff and $\Gamma_{k}^{(2)}$ is the Hessian of the EAA. To concretely employ equation (6) we will need to resort to some approximations and we will make an ansatz for $\Gamma_{k}$. Such procedure has been proved robust in many fields, especially to determine the scaling of statistical system at criticality, see 42 for an overview.

Equation (6) will provide the running for the couplings in our ansatz. Nevertheless we are also interested in the running of composite operators. To derive the flow equation for composite operators let us consider the one loop case. Besides the cutoff term let us add to the action a source $\varepsilon$ coupled to a composite operator $O$, then the one loop EAA reads

$$
\Gamma_{k, 1}=S+\varepsilon O+\frac{1}{2} \operatorname{Tr} \log \left(S^{(2)}+R_{k}+\varepsilon O^{(2)}\right)
$$

where $S^{(2)}$ and $O^{(2)}$ are the Hessians for the action and the composite operator respectively. If we now derive the above expression with respect to the scale $k$ we obtain:

$$
\partial_{t} \Gamma_{k, 1}=\frac{1}{2} \operatorname{Tr}\left(S^{(2)}+R_{k}+\varepsilon O^{(2)}\right)^{-1} \partial_{t} R_{k} .
$$

Finally, in order to derive the running of the composite operator, we just need to take a functional derivative with respect to the source $\varepsilon$ and set this to zero:

$$
\partial_{t} O=-\frac{1}{2} \operatorname{Tr}\left(S^{(2)}+R_{k}\right)^{-1} O^{(2)}\left(S^{(2)}+R_{k}\right)^{-1} \partial_{t} R_{k}
$$

The exact flow equation for the running of the composite operator can be found from the above expression just by "RG-improving" the one-loop case (exactly as it happens with the EAA). To see this explicitly we consider that, given a modified action $S+\varepsilon O$, both the connected Green's function generating functional $W_{k}[J, \varepsilon]$ and its Legendre transform $\tilde{\Gamma}_{k}[\varphi, \varepsilon]$ depend on the source $\varepsilon$. In particular from the definition of $\tilde{\Gamma}_{k}$ and $\Gamma_{k}$ we have

$$
\Gamma_{k}[\varphi, \varepsilon]=\int \varphi J-W_{k}[J, \varepsilon]-\Delta S_{k}[\varphi] .
$$

In order to extract information regarding the composite operator $O$ we simply take a functional derivative with respect to $\varepsilon$ and observe that

$$
\left.\frac{\delta}{\delta \varepsilon(x)} \Gamma_{k}[\varphi, \varepsilon]\right|_{\varepsilon=0}=-\left.\frac{\delta W_{k}[J, \varepsilon]}{\delta \varepsilon(x)}\right|_{\varepsilon=0} .
$$

This equation tells us that the running composite operator $[O]_{k}$ is directly related to the $\Gamma_{k}[\varphi, \varepsilon]$ via a functional derivative with respect to $\varepsilon$. The flow equation for $\Gamma_{k}[\varphi, \varepsilon]$ is simply given by equation (6) where the usual EAA is replaced with the $\varepsilon$-dependent EAA. Thus the running of the first functional derivative of $\Gamma_{k}[\varphi, \varepsilon]$ with respect to $\varepsilon$ can be directly obtained from (6) and, setting $\varepsilon=0$ at the end, one obtains:

$$
\begin{aligned}
\partial_{t} O_{k}=-\frac{1}{2} \operatorname{Tr}\left[\left(\Gamma_{k}^{(2)}+R_{k}\right)^{-1}\right. & O_{k}^{(2)} \\
& \left.\left(\Gamma_{k}^{(2)}+R_{k}\right)^{-1} \partial_{t} R_{k}\right] .
\end{aligned}
$$

Equation (7) will give back a differential equation to which a suitable boundary condition is associated. Typically one defines the composite operator of interest at some scale $\Lambda$. Clearly the RG evolution generally produces mixing among different operators and the composite operator has to be parametrized choosing a suitable ansatz. For a detailed discussion on the flow equation for composite operators we refer to [4].

Let us come back to the Kraichnan model. We choose the cutoff kernel $R_{k}$ as

$$
R_{k}=\left(\begin{array}{ccccc}
0 & R_{k, \theta \bar{\theta}} & 0 & 0 & 0 \\
R_{k, \bar{\theta} \theta} & 0 & 0 & 0 & 0 \\
0 & 0 & R_{k, v v} & 0 & 0 \\
0 & 0 & 0 & 0 & R_{k, c \bar{c}} \\
0 & 0 & 0 & R_{k, \bar{c} c} & 0
\end{array}\right) .
$$


The matrix acts on the following vector in field space: $(\theta, \bar{\theta}, v, c, \bar{c})$. As common in the application of FRG to non-equilibrium systems we have chosen an off-diagonal cutoff for the response field and its companion (e.g.: $\bar{\theta}$ and $\theta$ respectively). Moreover we do not cutoff the frequency since we will be able to perform integration over frequency analytically. Note that adding the cutoff may break some of the symmetries analysed in appendix $\mathrm{A}$. Of course at the fixed point the scaling behaviour does not depend on the form of the cutoff but breaking some symmetries in the middle of the flow means that more terms have to be taken into account. It is clear that if we consider

$$
R_{k}=\frac{1}{e^{\left(\frac{\Delta}{k^{2}}\right)^{\alpha}}-1}(\Delta)^{\alpha},
$$

where $\Delta=-\partial^{2}$, none of the symmetry is broken and in particular shift symmetry is preserved. If we chose the optimized cutoff 43

$$
R_{k}=\left(k^{2 \alpha}-\Delta^{\alpha}\right) \theta\left(k^{2}-\Delta\right)
$$

shift symmetry would be broken.

We are interested in the scaling of structure functions $S_{n}$ of the field $\theta$ at equal time, i.e.:

$$
S_{2 n} \equiv\left\langle(\theta(t, x)-\theta(t, y))^{2 n}\right\rangle .
$$

Clearly $S_{2 n}$ is given by a product of several different composite operators and to determine the scaling behaviour of $S_{2 n}$ is a rather difficult task. It is convenient to reason in terms of single composite operators expressing $S_{2 n}$ via the Operator Product Expansion (OPE), or Short Distance Expansion. Such program works nicely in the $\varepsilon$-expansion framework in 24] and we will follow closely this procedure. Here the main idea is to identify the operators which determine the scaling in such a way to be able to apply the FRG framework afterwards. First of all let us consider the simplest case of $S_{2}$ and recall that the canonical dimension of the field is $[\theta]=T^{1 / 2}$. For dimensional reason thus we have:

$$
\begin{aligned}
S_{2}= & T \cdot C_{1}\left(\mu_{T} T, \mu x\right)+C_{2}\left(\mu_{T} T, \mu x\right)\left[\theta^{2}\right] \\
& +T^{-1} C_{3}\left(\mu_{T} T, \mu x\right)\left[\theta^{4}\right]+\cdots+D_{1}\left(\mu_{T} T, \mu x\right) x^{2}[\partial \theta \partial \theta] \\
& +T^{-1} D_{2}\left(\mu_{T} T, \mu x\right) x^{4}\left[(\partial \theta \partial \theta)^{2}\right]+\cdots .
\end{aligned}
$$

Note that odd terms in $\theta$ are not allowed by $\mathbb{Z}_{2}$-symmetry. Moreover both the path integral and the correlation function in $S_{n}$ are shift invariant. As a consequence the operators entering in the OPE are also shift invariant, i.e.: only derivatives of $\theta$ appear and all the terms in the first line, except the first one, vanish. Finally let us recall that we are considering equal time correlators and so $T$ must be set to zero. Now, if we make the hypothesis that the time dependence of the various terms entering in (11) is not dramatically different from the canonical one, we can observe that for $T \rightarrow 0$ some terms vanish, some others stay finite and some diverge. We assume that the divergent terms are eliminated from the renormalization procedure so that only finite terms remain. At this point we note that only operators like $\partial \theta \partial \theta$ are relevant in (11), i.e.: operators which are not multiplied by overall factors of $T$. In full analogy one can argue that for $S_{2 n}$ the relevant composite operator is $(\partial \theta \partial \theta)^{2 n}$. This reasoning is at best heuristic and can be better justified by diagrammatic considerations as presented in 24] where it is shown that only composite operators with at most $2 n \theta$-fields appear in the OPE of $S_{2 n}$. Here our main message is that the OPE suggests that the scaling of the structure functions is encoded in the scaling of some particular composite operators whose leading term in a derivative expansion is $(\partial \theta \partial \theta)^{2 n}$. Note that this is the operator you get from a simple Taylor expansion of $S_{2 n}$.

In order to derive the formula for the anomalous dimension of a composite operator $O$ we consider the Callan-Symanzik equation and implement renormalization multipling the operator by a coupling $Z_{O}$. Let us consider the coupling appearing in $S_{\theta}$ and $S_{g}$, the CallanSymanzik equation reads:

$$
\left(\mu \partial_{\mu}+\beta_{\kappa} \partial_{\kappa}+\gamma_{O}\right)\langle O\rangle=0
$$

where we used the fact $D_{0}$ is not renormalized and thus does not enter in the equation and $\gamma_{O}=\partial_{t} Z_{O} / Z_{O}$. Moreover from dimensional analysis (in space and time) we have

$$
\begin{aligned}
& \left(-D_{0} \partial_{D_{0}}-\kappa \partial_{\kappa}+T \partial_{T}+d_{O}^{\omega}\right)\langle O\rangle=0 \\
& \left(-\mu \partial_{\mu}+(2-\varepsilon) D_{0} \partial_{D_{0}}\right. \\
& \left.\quad+2 \kappa \partial_{\kappa}+x \partial_{x}-M \partial_{M}+d_{O}^{k}\right)\langle O\rangle=0
\end{aligned}
$$

where $d_{O}^{\omega}$ and $d_{O}^{k}$ are the frequency and mass dimensions of the operator $O$. Now let us plug 12 in (14), we obtain

$$
\begin{aligned}
& \left(\beta_{\kappa} \partial_{\kappa}+\gamma_{O}+(2-\varepsilon) D_{0} \partial_{D_{0}}\right. \\
& \left.\quad+2 \kappa \partial_{\kappa}+x \partial_{x}-M \partial_{M}+d_{O}^{k}\right)\langle O\rangle=0 .
\end{aligned}
$$

Now we insert (13) in the above relation and find

$$
\begin{aligned}
& \left(\beta_{\kappa} \partial_{\kappa}+\gamma_{O}-\varepsilon D_{0} \partial_{D_{0}}+2 T \partial_{T}\right. \\
& \left.\quad+2 d_{O}^{\omega}+x \partial_{x}-M \partial_{M}+d_{O}^{k}\right)\langle O\rangle=0 .
\end{aligned}
$$

Finally it is convenient to re-express the flow via the coupling $g=D_{0} / \kappa$ and to observe that we can rewrite the beta function of $\kappa$ via $g$ exploiting the fact that $D_{0}$ is not renormalized:

$$
\begin{aligned}
\beta_{\kappa} \partial_{\kappa} & =\frac{\beta_{\kappa}}{\kappa} \kappa \partial_{\kappa} \\
& =\frac{k \frac{\partial}{\partial k}\left(\frac{\kappa}{D_{0}}\right)}{\frac{\kappa}{D_{0}}} \kappa \partial_{\kappa}=\frac{k \frac{\partial}{\partial k}\left(g^{-1}\right)}{g^{-1}} \kappa \partial_{\kappa} \\
& =-\frac{1}{g} \beta_{g} \kappa \partial_{\kappa} .
\end{aligned}
$$

Now we replace $\kappa \partial_{\kappa}$ using equation 13 and we express $\beta_{g}$ via its dimensionless counterpart $\tilde{g} \equiv g k^{-\varepsilon}$. Note that 
$\beta_{g}=\left(\varepsilon \tilde{g}+\beta_{\tilde{g}}\right) k^{\varepsilon}$ and so

$$
\begin{aligned}
\frac{1}{g} \beta_{g} \kappa \partial_{\kappa} & =\frac{1}{\tilde{g}}\left(\varepsilon \tilde{g}+\beta_{\tilde{g}}\right)\left(-D_{0} \partial_{D_{0}}+T \partial_{T}+d_{O}^{\omega}\right) \\
& =\varepsilon\left(-D_{0} \partial_{D_{0}}+T \partial_{T}+d_{O}^{\omega}\right)
\end{aligned}
$$

at a non-Gaussian fixed point. Now we plug this in equation (15):

$$
\begin{aligned}
& \left(-\varepsilon\left(-D_{0} \partial_{D_{0}}+T \partial_{T}+d_{O}^{\omega}\right)+\gamma_{O}-\varepsilon D_{0} \partial_{D_{0}}+2 T \partial_{T}\right. \\
& \left.+2 d_{O}^{\omega}+x \partial_{x}-M \partial_{M}+d_{O}^{k}\right)\langle O\rangle=0 \\
& \left((2-\varepsilon) T \partial_{T}+(2-\varepsilon) d_{O}^{\omega}\right. \\
& \left.\quad+x \partial_{x}-M \partial_{M}+d_{O}^{k}+\gamma_{O}\right)\langle O\rangle=0
\end{aligned}
$$

This equation will be used in section VI to derive the scaling of various composite operators and to determine the scaling of the structure functions. Summarizing we have introduced the FRG framework for the couplings and for the composite operators. Thus we used the OPE to identify which is the most important operator which contributes to the scaling of the structure function.

\section{THE IR FIXED POINT}

In this section we derive the IR fixed point whose scaling will be studied in the next sections. The presence of an IR fixed point for the Kraichnan model is known from $\varepsilon$-expansion in 24. In 24] it is argued that the beta function of $g$, which is the coupling we choose to parametrize the RG flow, is one loop exact. Thus we will first perform a one loop computation within the FRG framework and afterwards we will comment on how the fact that the beta function of $g$ is one loop exact can be understood from an FRG perspective.

We start by considering the ansatz:

$$
\begin{aligned}
\Gamma_{k}= & \int\left[\bar{\theta}\left(\partial_{t}+v^{i} \partial_{i}-\frac{\kappa}{2} \partial^{2}\right) \theta-\frac{1}{2} \bar{\theta} D_{\bar{\theta}} \bar{\theta}+\frac{1}{2} v D_{v}^{-1} v\right. \\
& \left.+\bar{c}\left(\partial_{t}+v^{i} \partial_{i}-\frac{\kappa}{2} \partial^{2}\right) c\right]
\end{aligned}
$$

In order to evaluate the running of $\kappa$ it is convenient to consider the flow equation for the functional derivatives of $\Gamma_{k}$. In particular we derive the flow equation (6) with respect to $\theta$ and $\bar{\theta}$ and extract the term proportional to the momentum square. The central object in the flow equation is the Hessian of the EAA which in field space and at zero field reads

$$
\Gamma_{k}^{(2)}=\left(\begin{array}{ccccc}
0 & \gamma_{\theta \bar{\theta}} & 0 & 0 & 0 \\
\gamma_{\bar{\theta} \theta} & \gamma_{\bar{\theta} \bar{\theta}} & 0 & 0 & 0 \\
0 & 0 & \gamma_{v v} & 0 & 0 \\
0 & 0 & 0 & 0 & -\gamma_{c \bar{c}} \\
0 & 0 & 0 & \gamma_{\bar{c} c} & 0
\end{array}\right)
$$

Thus the regularized propagator $G_{k}=\left(\Gamma_{k}^{(2)}+R_{k}\right)^{-1}$

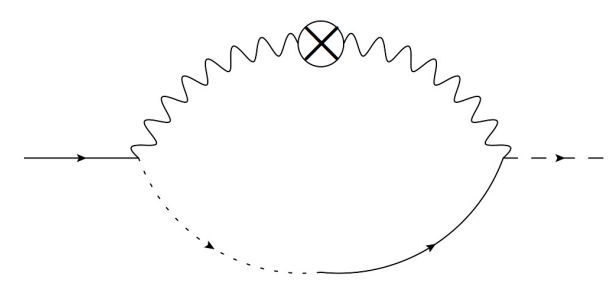

FIG. 1. Diagrammatic representation of the flow equation (19).

has the form

$$
G_{k}=\left(\begin{array}{ccccc}
G_{\bar{\theta} \bar{\theta}} & G_{\theta \bar{\theta}} & 0 & 0 & 0 \\
G_{\bar{\theta} \theta} & 0 & 0 & 0 & 0 \\
0 & 0 & G_{v v} & 0 & 0 \\
0 & 0 & 0 & 0 & G_{c \bar{c}} \\
0 & 0 & 0 & -G_{\bar{c} c} & 0
\end{array}\right) .
$$

In particular we have

$$
\begin{aligned}
G_{\theta \bar{\theta}} & =G_{\bar{\theta} \theta}^{*} \\
& =\frac{1}{i \omega+\frac{\kappa}{2} q^{2}+R_{k}} \\
G_{v v} & =\frac{D_{0}}{\left(q^{2}\right)^{\frac{d+\varepsilon}{2}}+R_{k}} P_{i j} \\
G_{c \bar{c}} & =G_{\bar{c} c}^{*} \\
& =\frac{1}{i \omega+\frac{\kappa}{2} q^{2}+R_{k}} .
\end{aligned}
$$

Schematically the flow equation for two point function has the following form:

$$
\begin{aligned}
\partial_{t} \frac{\delta^{2} \Gamma_{k}}{\delta \theta \delta \bar{\theta}}= & \frac{1}{2} \operatorname{Tr}\left[G_{k} \cdot \frac{\delta \Gamma_{k}^{(2)}}{\delta \theta} \cdot G_{k} \cdot \frac{\delta \Gamma_{k}^{(2)}}{\delta \bar{\theta}} \cdot G_{k} \cdot \partial_{t} R_{k}\right. \\
& +G_{k} \cdot \frac{\delta \Gamma_{k}^{(2)}}{\delta \bar{\theta}} \cdot G_{k} \cdot \frac{\delta \Gamma_{k}^{(2)}}{\delta \theta} \cdot G_{k} \cdot \partial_{t} R_{k} \\
& \left.-G_{k} \cdot \frac{\delta^{2} \Gamma_{k}^{(2)}}{\delta \theta \delta \bar{\theta}} \cdot G_{k} \cdot \partial_{t} R_{k}\right] .
\end{aligned}
$$

Note that the term in the third line does not contribute since there is no such vertex in the ansatz (17). Thus we find that

$$
\partial_{t} \frac{\delta^{2} \Gamma_{k}}{\delta \theta \delta \bar{\theta}}=G_{v v} \gamma^{(3)} G_{\theta \bar{\theta}} \gamma^{(3)} G_{v v} \partial_{t} R_{k, v v}
$$

where $\gamma^{(3)}$ denotes the vertex coming from the term $\bar{\theta} v^{i} \partial_{i} \theta$ in the action. Equation 19 can be written in a diagrammatic fashion as shown in Figure 1. Note that there are further contributions to the flow equation for the two point function but they vanish upon integration over the frequency (see appendix B for some details on 


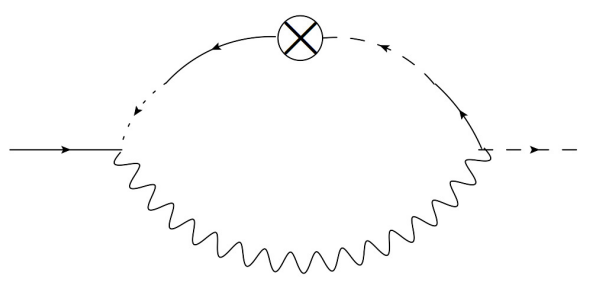

FIG. 2. Diagrammatic representation of the further contribution to the flow equation of $\kappa$ which vanish after frequency integration.

the frequency integration). These diagrams are shown in Figure 2. The final result is:

$$
\begin{gathered}
G_{v v} \gamma^{(3)} G_{\theta \bar{\theta}} \gamma^{(3)} G_{v v} \partial_{t} R_{k, v v}=-\frac{1}{2}\left(\frac{1}{(2 \pi)^{d}} \frac{2 \pi^{d / 2}}{\Gamma\left(\frac{d}{2}\right)} \frac{d-1}{d}\right) \frac{D_{0}}{2} \\
\cdot \int_{0}^{\infty} d z \frac{z^{d / 2-1}}{\left(z^{\frac{d+\varepsilon}{2}}+R_{k, v v}\right)^{2}} \partial_{t} R_{k, v v}
\end{gathered}
$$

If we use the exponential cutoff $(9)$ in the above equation we obtain

$$
\begin{aligned}
& G_{v v} \gamma^{(3)} G_{\theta \bar{\theta}} \gamma^{(3)} G_{v v} \partial_{t} R_{k, v v}=-\frac{1}{2}\left(\frac{1}{{(2 \pi)^{d}}^{d}} \frac{2 \pi^{d / 2}}{\Gamma\left(\frac{d}{2}\right)} \frac{d-1}{d}\right) \\
& \frac{D_{0}}{2} k^{-\varepsilon} \cdot\left(2 \frac{d+\varepsilon}{d} \Gamma\left[1+\frac{d}{d+\varepsilon}\right]\right)(21)
\end{aligned}
$$

and for the optimized cutoff 10

$$
\begin{aligned}
G_{v v} \gamma^{(3)} G_{\theta \bar{\theta}} \gamma^{(3)} G_{v v} \partial_{t} R_{k, v v}=- & \frac{1}{2}\left(\frac{1}{(2 \pi)^{d}} \frac{2 \pi^{d / 2}}{\Gamma\left(\frac{d}{2}\right)} \frac{d-1}{d}\right) \\
& \frac{D_{0}}{2} k^{-\varepsilon} \cdot\left(2 \frac{d+\varepsilon}{d}\right)(22)
\end{aligned}
$$

The fact that beta functions coming from $(21)$ and 22 . are slightly different has to be expected since the beta functions are scheme dependent and using different cutoffs corresponds to a change of scheme in the FRG language. Scheme independence has to be recovered in physical quantities like the critical exponents as we will check in a moment. In this case it is convenient to parametrize the flow via the coupling $g=D_{0} / \kappa$ and to introduce its dimensionless analogue $\tilde{g}=g k^{-\varepsilon}$. Note that the flow equation 20 has the following generic form

$$
\partial_{t}\left(\frac{\kappa}{2}\right)=-A D_{0} k^{-\varepsilon}
$$

where $A$ is a constant which depend on the dimension and on $\varepsilon$. In term of the dimensionless coupling $\tilde{g}$ the above equation reads:

$$
\partial_{t} \tilde{g}=-\varepsilon \tilde{g}+2 A \tilde{g}^{2} .
$$

The IR fixed point thus reads $\tilde{g}_{*}=\varepsilon /(2 A)$ and the critical exponent is $\beta_{\tilde{g}}^{\prime}=\varepsilon$ independently of the cutoff.

Let us compare our procedure with the one adopted in 24. In 24 the authors set up a field theoretic framework analogous to the one we have seen in section [II The only difference being the fact that the ghost fields are not present. The one loop correction to the molecular diffusivity is computed and the diagram is UV convergent. However when $\varepsilon \rightarrow 0$ a divergence appears. In full analogy with dimensional regularization the authors perform an $\varepsilon$-expansion and subtract the poles. This leads to a prescription for the RG flow which can be used to obtain a resummed result. When one performs this $\varepsilon$-expansion one notes that the scale $m$ disappears from the leading expression proportional to $1 / \varepsilon$ and disappears from the flow equations. In our case we simply set $m=0$ from the very beginning.

Note that in comparison to other works in the literature there are two main differences. First the velocity covariance (2) adopted in this work and in 24] differs from the one used in other works since the coefficient $D_{0}$ in (2) is replaced with $D_{0} \varepsilon$ (see for instance [5, 9, 10, 28]). Moreover the problem can be represented using the Ito discretization prescription in place of the Stratonovich one. With this definition the velocity covariance is finite in the limit $\varepsilon \rightarrow 0$ and the previously mentioned divergence for $\varepsilon \rightarrow 0$ is not present [28. In this case the RG flow of the action is trivial and non-trivial flows can be found for composite fields like $(\partial \theta)^{n}$. We refer to 28 for more details on the use of Ito prescription and the RG flows found in this case.

Finally in 24] it is argued that the beta function of $\tilde{g}$ is one-loop exact. To understand how this should be interpreted in the FRG scheme let us consider again the ansatz (17) and plug this in the flow equation for the two point function. We want to understand if further terms are generated by the RG flow or not. It is immediate to note that no further term is generated: the "trace factor" found by contracting the vertices with the propagator is $-P^{2}\left(1-X^{2}\right)$ and the propagator $G_{\theta \bar{\theta}}$ (which depends on $\left.p_{i}+q_{i}\right)$ loses its $P$-dependence after the frequency integration. As a consequence the only $P$-dependence comes from the trace factor. This means that the l.h.s. and r.h.s. of $\sqrt{19}$ are proportional to $P^{2}$, a very uncommon fact in FRG computations. One can be even more general and start from the following ansatz

$$
\begin{aligned}
\Gamma_{k}= & \int\left[\bar{\theta}\left(\partial_{t}+v^{i} \partial_{i}+\frac{\kappa}{2} f\left(-\partial^{2}\right)\right) \theta-\frac{1}{2} \bar{\theta} D_{\bar{\theta}} \bar{\theta}+\frac{1}{2} v D_{v}^{-1} v\right. \\
& \left.+\bar{c}\left(\partial_{t}+v^{i} \partial_{i}+\frac{\kappa}{2} f\left(-\partial^{2}\right)\right) c\right] .
\end{aligned}
$$

Repeating the same steps the flow equation itself tells that $f\left(-\partial^{2}\right)=-\partial^{2}$. We interpret this as an argument telling us that the beta function of $g$ is one-loop exact. Clearly one may consider even more general ansatzs which are consistent with all the symmetries we analyzed in appendix A. For example one can add higher powers 
in the fields via terms like:

$$
\bar{\theta}^{2} f\left(\nabla_{t},-\partial^{2}\right) \theta^{2}+2 \bar{\theta} \bar{c} f\left(\nabla_{t},-\partial^{2}\right) \theta c .
$$

We will not consider such terms but these terms should be taken into account in a more complete computation.

\section{RG FLOW OF COMPOSITE OPERATORS}

In this section we study the scaling of the structure function at the IR fixed point found in section IV We will first study the renormalization of the operator $(\partial \theta \partial \theta)^{n}$ and then we will move to consider $\theta^{m}$. The results found in this section will be used in section VI to obtain the correction to the scaling of $S_{2 n}$.

\section{A. The operator $(\partial \theta \partial \theta)^{n}$}

In section III we argued that the scaling of the structure functions $S_{2 n}$ is encoded in the scaling of the composite operator $(\partial \theta \partial \theta)^{n}$. The argument is based on the OPE of $S_{2 n}$. Let us stress that if we take into account only the operator $(\partial \theta \partial \theta)^{n}$ we are making an approximation since other operators also contribute, e.g.: $\left(\partial^{2} \theta \partial^{2} \theta\right)^{n}$. In this sense we apply the logic of the derivative expansion to the composite operator coming from the OPE of $S_{2 n}$.

From now on we focus on the RG flow of the operator $O=(\partial \theta \partial \theta)^{n}$. To compute its running we introduce a coupling $Z_{O}$ which multiplies $O$ and use equation (7). To extract the running of $Z_{O}$ we perform $2 n$ functional derivatives with respect to $\theta$ and set all the fields to zero. Thus l.h.s. is proportional to $P^{2 n}$ and has to be compared with the terms also proportional to $P^{2 n}$ obtained by taking $2 n$ functional derivatives of the r.h.s. of 77 . Note that the Hessian of the operator $O$ appears in equation (7) and $2 n-2$ functional derivatives will act on it while the remaining two will act on the regularized propagators of the EAA. Moreover the l.h.s. of the equation is proportional to $\partial_{t} Z_{O}$ while the r.h.s. to $Z_{O}$ so it will be easy to extract the anomalous dimension $\partial_{t} Z_{O} / Z_{O}$. The flow equation obtained in this way can be represented diagrammatically as in Figures 3, 4 and 5 where the black dot denotes the insertion of the Hessian of the composite operator with $2 n-2$ functional derivatives attached.

We limit ourselves to consider the leading term in the $\varepsilon$-expansion of the structure function which amounts to a one loop computation. Our main objective is to test the tools introduced so far and reproduce the known leading correction. In each diagram the "trace factor" between the vertices and the velocity projector gives $P^{2}\left(1-X^{2}\right)$, where $X$ is the cosine between the external and the loop momentum. The Hessian of the composite operator reads $O^{(2)}=2 n P^{2(n-1)} Q^{2}\left[1+X^{2} 2(n-1)\right]$, where $P$ and $Q$ are the modulus of the external and loop momentum respectively. In each diagram the product of the "trace

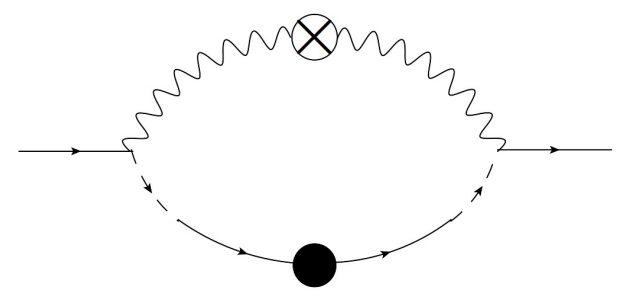

FIG. 3. Diagrammatic representation of a contribution to flow equation for composite operators.

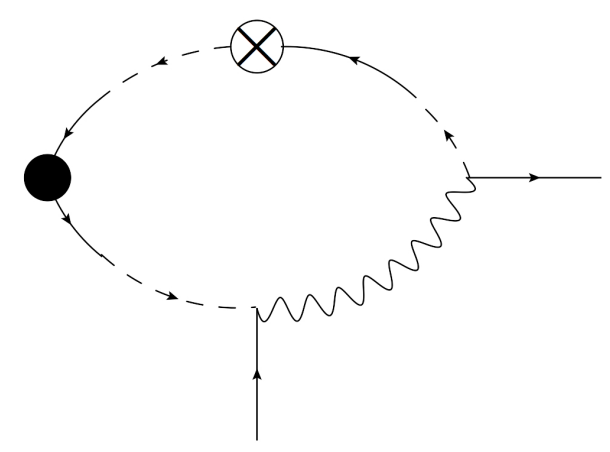

FIG. 4. Diagrammatic representation of a contribution to flow equation for composite operators.

factor" and of $O^{(2)}$ gives a term of order $P^{2 n}$. Thus neglecting all the $P$-dependence in the regularized propagators we obtain the running of $Z_{O}$ and the angular integral factors out. The contribution to $\partial_{t} Z_{O} / Z_{O}$ from

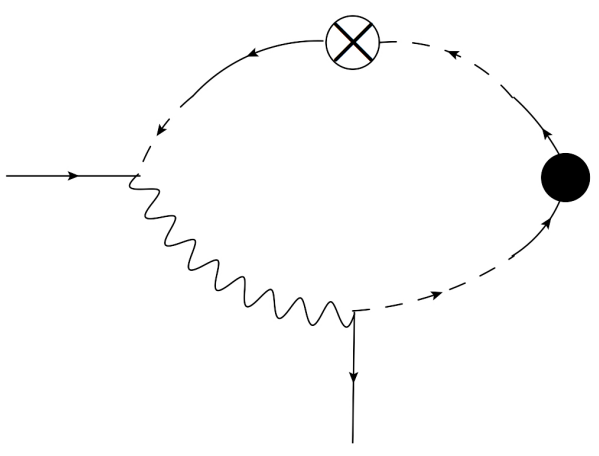

FIG. 5. Diagrammatic representation of a contribution to flow equation for composite operators. 
the diagram of Figure 3 is

$$
\begin{aligned}
& -\frac{2^{-d} \pi^{-d / 2}}{\Gamma\left(\frac{d}{2}\right)} \frac{d-1}{d}\left(n \frac{d+2 n}{d+2}\right) \frac{D_{0}}{\kappa} \\
& \int_{0}^{\infty} d z z^{d / 2} \frac{1}{z+R_{k, \theta \bar{\theta}}} \frac{1}{\left(z^{\frac{d+\varepsilon}{2}}+R_{k, v v}\right)^{2}} \partial_{t} R_{k, v v} .
\end{aligned}
$$

Furthermore both the diagrams of Figures 4 and 5 give the following contribution to $\partial_{t} Z_{O} / Z_{O}$ :

$$
\begin{aligned}
& -\frac{2^{-d} \pi^{-d / 2}}{\Gamma\left(\frac{d}{2}\right)} \frac{d-1}{d}\left(n \frac{d+2 n}{d+2}\right) \frac{1}{2} \frac{D_{0}}{\kappa} \\
& \int_{0}^{\infty} d z z^{d / 2} \frac{1}{z^{\frac{d+\varepsilon}{2}}+R_{k, v v}} \frac{1}{\left(z+R_{k, \theta \bar{\theta}}\right)^{2}} \partial_{t} R_{k, \theta \bar{\theta}} .
\end{aligned}
$$

The integrals can be solved analytically employing the optimized cutoff (10) and, summing all the contributions, the leading contribution for small $\varepsilon$ is

$$
\frac{\partial_{t} Z_{O}}{Z_{O}}=-\frac{2^{1-d} \pi^{-d / 2}}{\Gamma\left(\frac{d}{2}\right)} \frac{d-1}{d}\left(n \frac{d+2 n}{d+2}\right) \tilde{g} .
$$

At the leading order in $\varepsilon$ we have:

$$
\tilde{g}_{*}=\frac{d}{d-1}\left[\frac{1}{(2 \pi)^{d}}\left(\frac{2 \pi^{d / 2}}{\Gamma\left(\frac{d}{2}\right)}\right)\right]^{-1} \varepsilon .
$$

Therefore we obtain

$$
\left.\frac{\partial_{t} Z_{O}}{Z_{O}}\right|_{\tilde{g}_{*}}=-\left[n \frac{d+2 n}{d+2}\right] \varepsilon .
$$

Although this computation is shown using the optimized cutoff we checked that the fixed point anomalous dimension above is very stable under changes of the cutoff kernel $R_{k}$.

Let us note that differently from what we have seen in section IV] the r.h.s. of the flow equation for composite operators generates new terms. Indeed it is possible to verify that terms of higher power in $P$ are generated expanding those propagators which are function of $\left(p_{i}+q_{i}\right)$. This means that, within our approximation, the system is not closed. This is a common problem in the FRG approach and the crucial point is to parametrize the quantities of interest with sufficiently many terms. Here, retaining just one operator, we did a rather crude approximation and this precludes for instance the possibility of extracting correct values of the anomalous dimension for large values of $\varepsilon$. However we stress that hopefully such difficulties can be solved by employing larger truncations as usually happens in the FRG applications. In order to understand if this methodology is efficient for the Kraichnan model one should increase the truncation and check the stability of the results. This however is beyond the scope of the present work.

\section{B. The operator $\theta^{m}$}

Besides the anomalous dimension of the operator $(\partial \theta \partial \theta)^{n}$ it is useful to consider also the anomalous dimension of operators $\theta^{m}$. It turns out that the anomalous of $\theta^{m}$ dimension is zero. To see this we consider the flow equation for the composite operator $\theta^{m}$ which is fully analogous to the one for $(\partial \theta \partial \theta)^{n}$. One only needs to change the "composite operator vertex" in the diagrams. This vertex is simply given by $m(m-1)$ (from the Hessian of $\theta^{m}$ ). Now we recall that the trace over the vertices and the projectors in these diagrams gives an overall factor of $P^{2}$. Since the definition of the running of $Z_{\theta^{m}}$ is taken at zero momentum we see that the r.h.s. of the flow equation for $Z_{\theta^{m}}$ is zero.

As an aside comment let us note that a non-trivial result is found considering the Kraichnan model for a compressible fluid as done in 44. In this case the action reads

$S_{\theta}=\int\left[\bar{\theta}\left(\partial_{t} \theta+\partial_{i}\left(v^{i} \theta\right)-\frac{\kappa}{2} \partial^{2} \theta\right)-\frac{1}{2} \bar{\theta} D_{\bar{\theta}} \bar{\theta}+\frac{1}{2} v D_{v}^{-1} v\right]$

with

$$
D_{v}=\frac{D_{0}}{(2 \pi)^{d}} \delta\left(t-t^{\prime}\right) \int d^{d} k \frac{P_{i j}^{T}+\alpha P_{i j}^{L}}{\left(k^{2}+m^{2}\right)^{\frac{d}{2}+\frac{\varepsilon}{2}}} e^{i k x_{1}-i k x_{2}},
$$

where $P_{i j}^{T}$ and $P_{i j}^{L}$ are the transverse and longitudinal projectors respectively. This model has been studied in [44] and we have checked at one loop that the fixed point and the anomalous dimensions $\partial_{t} Z_{\theta^{m}} / Z_{\theta^{m}}$ are reproduced by our framework.

\section{SCALING OF THE STRUCTURE FUNCTIONS}

We use the results of sections IV and $\mathrm{V}$ in order to derive the running of the structure functions. To achieve this we will consider the OPE of $S_{2 n}$ [24]. Let us note that

$$
\begin{aligned}
S_{2 n} & =\left\langle(\theta(x)-\theta(y))^{2 n}\right\rangle \\
& =\sum_{k=0}^{2 n}\left(\begin{array}{c}
2 n \\
k
\end{array}\right)\left\langle\theta(x)^{2 n-k} \theta(y)^{k}\right\rangle .
\end{aligned}
$$

Repeating the same steps which lead to equation $(16)$ we obtain that all the terms in the above sum satisfy the following equation

$$
\left[r \partial_{r}+(2-\varepsilon) T \partial_{T}-n(2-\varepsilon)-M \partial_{M}\right]\left\langle\theta^{2 n-k} \theta^{k}\right\rangle=0
$$

where $r=|x-y|$. This tells us that

$$
S_{2 n} \sim r^{n(2-\varepsilon)} f(M r) .
$$

In order to understand the $M$-dependence in $S_{2 n}$ we consider the OPE. In section III we argued that the leading term in the OPE of $S_{2 n}$ is given by $(\partial \theta \partial \theta)^{n}$ thus

$$
S_{2 n} \approx\left\langle C(r)(\partial \theta \partial \theta)^{n}\right\rangle
$$


where $C(r)$ is the first Wilson coefficient of the expansion, a function of $(x-y) / 2$, and $(\partial \theta \partial \theta)^{n}$ is a composite operator in $(x+y) / 2$. Thus we have

$$
S_{2 n} \approx C(r)\left\langle(\partial \theta \partial \theta)^{n}\right\rangle
$$

where $\left\langle(\partial \theta \partial \theta)^{n}\right\rangle$ is a constant in the sense that it does not dependent on any coordinates. Now we consider equation (16) for the above correlation and deduce that

$$
\left(-M \partial_{M}+n \varepsilon+\gamma_{O}^{*}\right)\left\langle(\partial \theta \partial \theta)^{n}\right\rangle=0
$$

where we used the fact that $\left\langle(\partial \theta \partial \theta)^{n}\right\rangle$ does not depend on any coordinates. This tells us that

$$
\left\langle(\partial \theta \partial \theta)^{n}\right\rangle \sim M^{n \varepsilon+\gamma_{O}^{*}} .
$$

Finally we suppose that the Wilson coefficient $C(r)$ does not depend on $M$ in the limit $M \rightarrow 0$, i.e.: that $C(r)$ is regular in $M$. Note that the limit $M \rightarrow 0$, i.e.: $L_{F} \gg r$, is justified by the fact that we are studying the inertial regime of the system. This tells us that the whole $M$-dependence in $S_{2 n}$ is given by expression (23). Therefore we conclude that

$$
S_{2 n} \sim r^{n(2-\varepsilon)}(M r)^{n \varepsilon+\gamma_{O}^{*}}
$$

where

$$
n \varepsilon+\gamma_{O}^{*}=-\frac{2 n(n-1)}{d+2} \varepsilon .
$$

This is the leading correction found in [5, 6, via the zero mode approach and in 24] via the $\varepsilon$-expansion.

Let us note that we can derive the scaling of $S_{2 n}$ also via the following reasoning. Since $\left\langle(\partial \theta \partial \theta)^{n}\right\rangle$ does not depend on $r$ we can limit ourselves to study the $r$-dependence in $S_{2 n}$ studying the Wilson coefficient $C(r)$. We suppose that $C(r)$ is regular in $M$ and consider the $M \rightarrow 0$ limit. The Callan-Symanzik equation for the Wilson coefficient is:

$$
\left(\mu \partial_{\mu}+\beta_{g} \partial_{g}-\gamma_{O}\right) C=0
$$

where we used the fact that the anomalous dimension of the composite operators $\theta^{2 n-k}$ and $\theta^{k}$ are zero. Once again we repeat the steps which lead to equation (16) and obtain

$$
\left(r \partial_{r}-2 n+(2-\varepsilon) T \partial_{T}-\gamma_{O}^{*}-M \partial_{M}\right) C=0 .
$$

Thus we obtain

$$
C(r) \sim r^{2 n+\gamma_{O}^{*}}
$$

where the exponent is exactly the one coming from the sum of the exponents in 24.

\section{SUMMARY AND OUTLOOKS}

We have explored the application of the FRG framework to the Kraichnan model. In particular we studied the scaling of the structure functions in the Kraichnan model and reproduced the leading corrections by means of FRG techniques. From the field theoretical point of view the structure functions are correlations of composite operators. Thus in section III we recalled how to compute the running of composite operators using the flow equation for the EAA. In section [V] and $[\mathrm{V}$ we studied the IR fixed point of the model and the anomalous dimensions of various composite operators at the fixed point. The scaling of the structure functions in the Kraichnan model has been found in section VI using the OPE.

The path integral formulation adopted in this work has been explained in section [II while the associated symmetries and some non-renormalization theorems are discussed in appendix A. In the path integral formulation of the problem a determinant naturally arises and we kept track of this by expressing the determinant via the integration over some ghost fields. The ghost sector carries two new symmetries which we called BRS symmetry (mixing $\theta$ and $\bar{c}$ ) and ghost symmetry. These symmetries are very similar to the ones present in the path integral formulation for classical mechanics [30]. In the latter case more symmetries are present, this is due to the fact that the equations of motion for the Kraichnan model are not Hamiltonian. Let us note that the Navier-Stokes equation can be formulated as Hamiltonian equations of motion setting the viscosity to zero and using the so called Clebsh variables. It may turn out interesting to study the role of the symmetries and of the RG flow in this context. Moreover, as far as classical mechanics is concerned, the ghost fields carry information on the Lyapunov exponents of the system and thus on the chaoticity/stochasticity of the system [31 and we feel that they could play a role also in the path integral approach to turbulence. Here we limit ourselves to note that in the Kraichnan model the equation of motion for the ghost $c$ corresponds to unforced solutions which are the ones involved in the zero mode approach. We hope to study these topics in the future.

Finally we would like to stress that the techniques adopted in this work can be applied to more complicated systems, for instance the randomly stirred Navier-Stokes equation. On general grounds this will require to fully exploit the functional character of the flow equation in order to be able to account for an infinite dimensional ansatz and not to rely on the derivative expansion. This has been achieved successfully in the case of the KardarParisi-Zhang equation in 21]. In the Kraichnan model the situation is somehow simpler since the IR fixed point is perturbative for small $\varepsilon$ and the beta function of $g$ is one loop exact [24]. Nevertheless we believe that the logic adopted here, especially with regards to composite operators, can be used in other contexts and more work is needed in this direction. The advantage of the FRG framework is that it does not rely on a perturbative expansion and that genuinely non-perturbative results can be obtained provided that the EAA is parametrized with sufficient accuracy. In particular we hope that a similar framework to the one adopted in this work may be 
applied to more complicated situations, like the NavierStokes equation.

\section{ACKNOWLEDGMENTS}

The author acknowledges the support of the Foundation Blanceflor Boncompagni Ludovisi, née Bildt. The author is grateful to Martin Reuter for helpful discussions.

\section{Appendix A: symmetries}

In this appendix we consider the symmetries associated to the Krainchnan model. We first consider the symmetries associated to the action $S_{\theta}$ which can be easily extended to the ghost action. Finally we consider symmetries coming from non trivial cancellation in $S_{\theta}+S_{g h}$. From these symmetries we will be able to derive some non-renormalization theorems. Such program has been carried out in great detail for the Kardar-Parisi-Zhang equation in 21] and more recently for the Navier-Stokes equation in 19. In these cases the analysis showed that symmetries entail important constraints on the RG flow. Here we perform a similar analysis also including the ghost sector.

The action is symmetric under a sort of $\mathbb{Z}_{2}$ transformation: $\theta \rightarrow-\theta$ and $\bar{\theta} \rightarrow-\bar{\theta}$. This tells us that there is an even number of $\theta$ and $\bar{\theta}$ fields in any monomial of the effective action. Moreover it is easy to verify the invariance under constant shift in $\theta$

$$
\theta \rightarrow \theta+u
$$

Clearly this property is enjoyed also by the effective action. In analogy with what has been done in 21] we consider a time dependent shift:

$$
\theta \rightarrow \theta+u(t)
$$

This is not a symmetry but the bare action is noninvariant just by a term which is linear in field space. In particular the only time derivative appears in

$$
\bar{\theta} \partial_{t} \theta \rightarrow \bar{\theta} \partial_{t} \theta+\bar{\theta} \partial_{t} u(t)
$$

Since the non-invariance of the action is given just by a term which is linear in the field we can derive the following Ward Idendity (WI) [

$$
\begin{aligned}
0 & =\left\langle J \cdot u(t)-\int \bar{\theta} \partial_{t} u(t)\right\rangle \\
& =\left\langle\left(\frac{\delta \Gamma}{\delta \theta} \cdot u(t)-\int \bar{\theta} \partial_{t} u(t)\right)\right\rangle .
\end{aligned}
$$

\footnotetext{
${ }^{1}$ We use the following conventions: $\exp W=\int \exp [-S+J \cdot \phi]$ and $\Gamma=\int J \varphi-W$. Thus $J=\delta_{\varphi} \Gamma$.
}

This equation tells us that the term in the bare action $\int \bar{\theta} \partial_{t} \theta$ does not get renormalized. Moreover the action and the effective action also enjoy Galilei invariance as we will see in a moment. Thus we can infer that the term $\int \bar{\theta}\left(\partial_{t}+v^{i} \partial_{i}\right) \theta$ is not renormalized.

We also consider a time dependent shift in the response field $\bar{\theta} \rightarrow \bar{\theta}+\varepsilon(t)$ and we have

$$
\begin{aligned}
0 & =\left\langle-\int \varepsilon(t)\left(\partial_{t} \theta\right)+\varepsilon(t)\langle f f\rangle \bar{\theta}+J_{\bar{\theta}} \varepsilon_{t}\right\rangle \\
-\frac{\delta \Gamma}{\delta \bar{\theta}} \cdot \varepsilon_{t} & =-\int \varepsilon(t)\left(\partial_{t} \theta\right)+\varepsilon(t)\langle f f\rangle \bar{\theta} .
\end{aligned}
$$

This entails the non-renormalization of the term $\frac{1}{2} \bar{\theta}\langle f f\rangle \bar{\theta}$.

Now we consider the Galilei invariance of the model, the transformation is:

$$
\begin{aligned}
v(t, x) & \rightarrow v^{\prime}\left(t, x^{\prime}\right)+c=v^{\prime}(t, x-c t)+c \\
x & \rightarrow x^{\prime}=x-c t \\
t & \rightarrow t^{\prime} \\
\bar{\theta}(t, x) & \rightarrow \bar{\theta}\left(t, x^{\prime}\right) \\
\theta(t, x) & \rightarrow \theta\left(t, x^{\prime}\right) .
\end{aligned}
$$

This invariance tells us that time derivative actually appears via Galilei covariant derivative of the form $\partial_{t}+v^{i} \partial_{i}$. Note that the term quadratic in the velocities is not straightforwardly invariant. This is due to the fact that the average velocity is set to zero, in order to see directly Galilei invariance we must express the kinetic term as $(v-\langle v\rangle) D_{v}(v-\langle v\rangle)$. More precisely it turns out very useful also to consider the following time gauged Galilei transformation:

$$
\begin{aligned}
v(t, x) & \rightarrow v^{\prime}\left(t, x^{\prime}\right)+c(t)=v^{\prime}(t, x-c(t))+\dot{c}(t) \\
x & \rightarrow x^{\prime}=x-c(t) \\
t & \rightarrow t^{\prime} \\
\bar{\theta}(t, x) & \rightarrow \bar{\theta}\left(t, x^{\prime}\right) \\
\theta(t, x) & \rightarrow \theta\left(t, x^{\prime}\right) .
\end{aligned}
$$

We observe that

$$
\begin{aligned}
\left(\partial_{t}+v \partial_{x}\right) \theta \rightarrow & {\left[\partial_{t} \theta_{t, x-c(t)}+\partial_{x} \theta_{t, x-c(t)} \cdot\left(-\partial_{t} c\right)\right] } \\
& +\left(v_{t, x-c(t)}+\partial_{t} c(t)\right) \partial_{x} \theta_{t, x-c(t)} \\
= & {\left[\left(\partial_{t}+v \partial_{x}\right) \theta\right]^{\prime} . }
\end{aligned}
$$

Note that this is a symmetry of the equation (1) itself which is not preserved by the averaging over the velocities. Indeed performing a transformation in the path integral we obtain

$$
0=\left\langle-\int v D_{v} \delta v+J_{v} \delta v\right\rangle .
$$

This entails the non-renormalization of the "kinetic" term for the velocity fields. Thus we observe that the effective action is invariant under the time-gauged version of the Galilei transformations except for the quadratic term in the velocity field. All these non-renormalization 
theorems are known in the perturbative framework, see [24.

So far we have been considering symmetries which do not rely on the presence of the ghost. Indeed $S_{\theta}$ and $S_{g h}$ are both simultaneously invariant. Now we examine symmetries which exploit a non-trivial cancellation between $S_{\theta}$ and $S_{g h}$. Let us note that the action can be rewritten as

$$
S=-\frac{1}{2} \bar{\theta} D_{\bar{\theta}} \bar{\theta}+\bar{\theta} O_{v} \theta+\bar{c} O_{v} c+\frac{1}{2} v D_{v}^{-1} v
$$

where $O_{v} \equiv \partial_{t}+v^{i} \partial_{i}-\frac{\kappa}{2} \partial^{2}$. It is easy to check that $\theta \rightarrow \theta+\varepsilon c, \bar{c} \rightarrow \bar{c}-\varepsilon \bar{\theta}$ is a symmetry which we call BRS symmetry in analogy to nomenclature in [30]. This symmetry has been discussed in 36] and we briefly review it here. The symmetry transformation is linear and thus the BRS invariance is present also in the effective action. To derive some useful results we can consider $\left\langle\delta_{B R S} \mathcal{O}\right\rangle=0$ for some particular operators. For instance we can consider $\langle\delta(\bar{c} \theta)\rangle=\langle(-\bar{\theta} \theta+\bar{c} c)\rangle=0$ and $\langle\delta(\bar{c} \bar{\theta})\rangle \sim\langle\bar{\theta} \bar{\theta}\rangle=0$. Finally we have also the following symmetry: $\bar{c} \rightarrow \bar{c}-\varepsilon \bar{c}$ and $c \rightarrow c+\varepsilon c$. This symmetry entails the ghost number conservation.

\section{Appendix B: some frequency integrals}

In this appendix we provide a list of the typical frequency integral we have encountered. These integral come from the multiplication of various $\theta \bar{\theta}$-propagator entering in each diagram. The regularized propagator has the following form:

$$
\frac{1}{ \pm i \omega+V}=\frac{\mp i \omega+V}{\omega^{2}+V^{2}} .
$$

First of all we consider

$$
G(q) \equiv \frac{1}{2 \pi} \int d \omega\left(-i \omega+\nu q^{2}\right)^{-1}
$$

This integral is divergent but its principal value is well defined:

$$
\begin{aligned}
\frac{1}{2 \pi} \mathcal{P}\left[\int d \omega(-i \omega+V)^{-1}\right] & =\frac{1}{2 \pi} \mathcal{P}\left[\int d \omega \frac{i \omega+V}{\omega^{2}+V^{2}}\right] \\
& =\frac{1}{2} .
\end{aligned}
$$

Moreover we need

$$
\begin{aligned}
\frac{1}{2 \pi} \int\left(\frac{1}{ \pm i \omega+V}\right)^{2} & =0 \\
\frac{1}{2 \pi} \int d \omega \frac{1}{i \omega+V} \frac{1}{-i \omega+V} \frac{1}{i \omega+V} & =\frac{1}{4} \frac{1}{V^{2}} .
\end{aligned}
$$

The first integral above is somewhat unusual in the this type of computations, since one typically finds

$$
\frac{1}{2 \pi} \int \frac{1}{-i \omega+V} \cdot \frac{1}{i \omega+V}=\frac{1}{2 V} .
$$

This happens because our diagrams contains not only $\theta \bar{\theta}$-propagator but also velocity propagators (which are delta in time).
[1] A. N. Kolmogorov, C.R. Acad. Sci. URSS 30, 301-305 (1941).

[2] R. H. Kraichnan, Phys. Rev. Lett. 72, 1016 (1994).

[3] S. Corssin, J. Appl. Phys. 22, 469 (1951).

[4] A. Obukhov, Izv. Akad. Naut. SSSR, Ser. Geogr. I. Geofiz. 13, 55 (1949).

[5] K. Gawedzki and A. Kupiainen, Phys. Rev. Lett. 75, 3834 (1995).

[6] M. Chertkov and G. Falkovich, Phys. Rev. Lett. 76, 2706 (1996) chao-dyn/9509007.

[7] M. Chertkov, G. Falkovich, I. Kolokolov and V. Lebedev, Phys. Rev. E 52, 4924 (1995).

[8] B. I. Shraiman and E. D. Siggia, Comptes Rendus de l'Académie des Sciences Sries II b 321, 279 (1995).

[9] D. Bernard, K. Gawedzki and A. Kupiainen, Phys. Rev. E 54, 2564 (1996) chao-dyn/9601018.

[10] D. Bernard, K. Gawedzki and A. Kupiainen, Jour. of Stat. Phys. 90, 519, (1998) cond-mat/9706035.

[11] G. Falkovich, K. Gawedzki and M. Vergassola, Rev. Mod. Phys. 73, 913 (2001).

[12] J. Berges and D. Mesterhazy, Nucl. Phys. Proc. Suppl. 228, 37 (2012) arXiv:1204.1489 [hep-ph]].

[13] L. Canet, H. Chate and B. Delamotte, J. Phys. A 44, 495001 (2011) arXiv:1106.4129 [cond-mat.stat-mech]].

[14] C. Wetterich, Phys. Lett. B 301, 90 (1993).
[15] U. Ellwanger, Z. Phys. C 62, 503 (1994) hep$\mathrm{ph} / 9308260$.

[16] T. R. Morris, Int. J. Mod. Phys. A 9, 2411 (1994) hep$\mathrm{ph} / 9308265$.

[17] R. Collina and P. Tomassini, Phys. Lett. B 411, 117 (1997) hep-th/9709185.

[18] C. Mejía-Monasterio and P. Muratore-Ginanneschi, Phys. Rev. E 86, 016315 (2012). arXiv:1202.4588.

[19] L. Canet, B. Delamotte and N. Wschebor, arXiv:1411.7778 [cond-mat.stat-mech].

[20] L. Canet, B. Delamotte and N. Wschebor, arXiv:1411.7780 [cond-mat.stat-mech].

[21] L. Canet, H. Chate, B. Delamotte and N. Wschebor, Phys. Rev. E 84, 061128 (2011) arXiv:1107.2289 [condmat.stat-mech]].

[22] T. Kloss, L. Canet, B. Delamotte and N. Wschebor, Phys. Rev. E 89, no. 2, 022108 (2014) arXiv:1312.6028 [cond-mat.stat-mech]].

[23] S. Mathey, T. Gasenzer and J. M. Pawlowski, arXiv:1405.7652 [cond-mat.quant-gas].

[24] L. Ts. Adzhemyan, A. V. Antonov and A. N. Vasil'ev, Phys. Rev. E 58, 1823 (1998), chao-dyn/9801033.

[25] L. Ts. Adzhemyan, N. V. Antonov, and A. N. Vasil'ev, Usp. Fiz. Nauk, 166, 1257 (1996) [Phys. Usp. 39, 1193 (1996)]. 
[26] L.Ts. Adzhemyan, A.V. Antonov and A.N. Vasil'ev, "The field theoretic renormalization group in fully developed turbulence", Gordon and Breach, Amsterdam (1999).

[27] L.Ts. Adzhemyan, A.V. Antonov, V.A. Barinov, Yu.S. Kabrits and A.N. Vasil'ev, Phys. Rev. E 63, 025303(R) (2001), erratum E 64 019901, arxiv:nlin.CD/0010031

[28] A. Kupiainen and P. Muratore-Ginanneschi, Jour. of Stat. Phys., 126, 669 (2007); nlin/0603031 [nlin.CD].

[29] E. Gozzi, Phys. Rev. D 28, 1922 (1983).

[30] E. Gozzi, M. Reuter and W. D. Thacker, Phys. Rev. D 40, 3363 (1989).

[31] E. Gozzi and M. Reuter, Chaos Solitons Fractals 4, 1117 (1994).

[32] B. Sakita, "Quantum theory of many variable systems and fields," World Sci. Lect. Notes Phys. 1, 1 (1985).

[33] P. C. Martin, E. D. Siggia and H. A. Rose, Phys. Rev. A 8, 423 (1973).

[34] H. K. Janssen, Z. Phys. B: Cond. Mat. 23, 377 (1976).
[35] C. de Dominicis, J. Phys. Colloques 37, 247 (1976).

[36] G. Munoz and W. S. Burgett, Jour. of Stat. Phys. 56, 59 (1989), JHU-TIPAC-8906.

[37] T. R. Morris, Prog. Theor. Phys. Suppl. 131, 395 (1998) hep-th/9802039.

[38] C. Bagnuls and C. Bervillier, Phys. Rept. 348, 91 (2001) hep-th/0002034.

[39] J. Berges, N. Tetradis and C. Wetterich, Phys. Rept. 363, 223 (2002) hep-ph/0005122.

[40] M. Niedermaier and M. Reuter, Living Rev. Rel. 9, 5 (2006).

[41] Y. Igarashi, K. Itoh and H. Sonoda, Prog. Theor. Phys. Suppl. 181, 1 (2010) arXiv:0909.0327 [hep-th]].

[42] B. Delamotte, Lect. Notes Phys. 852, 49 (2012) condmat/0702365 [COND-MAT]].

[43] D. F. Litim, Phys. Rev. D 64, 105007 (2001) hepth/0103195.

[44] L. Ts. Adzhemyan and A. V. Antonov, Phys. Rev. E 58, 7381 (1998), chao-dyn/9806004 